\title{
INTERACTIVE ILUSTRATION FOR PHOTOSYNTHESIS TEACHING
}

\author{
Pereira, M.R.; Dórea, F.C.; Lima, C.M.R.; Castro, M.S. and Fontes, W.
}

Laboratório de Bioquímica e Química de Proteínas, Departamento de Biologia Celular, Universidade de Brasília, Brasília/DF

Computational resources became the major tool in the challenge of making high education more easy and motivating. Complex Biochemical pathways can now be presented in interactive and threedimensional animations. One of the most complex (detailed) and interesting metabolic pathway that students must understand in biochemical courses is photosynthesis. The light-dependent reactions are of special interest since they involve many different kinds of mechanisms, as light absorption by membrane complexes, proteins movement inside membranes, reactions of water hydrolysis, and electrons flow; making it difficult to understand by static bi-dimensional representations.

The resources of animation and ActionScript programming were used to make an interactive animation of photosynthesis, which at some times even simulates three-dimensionality. The animation begins with a leaf and progressively zooms in, until we have a scheme of a tylakoyd membrane, where each of the different steps of the pathway can be clicked to reveal a more detailed scheme of it. Where appropriate, the energy graphs are shown side by side with the reactions. The electron is represented with a face, so it can be shown to be stressing while going up in the energy graphs. Finally, there is a simplified version of the whole pathway, to illustrate how it all goes together.

The objective is to help professors on teaching the subject in regular classes, since currently all the explanations are omitted. In a future version, texts will be added to each step so it can be self-explicative to the students, helping them even on home or on-line learning.

Support: FUB/UnB; CNPq/PIBIC; MEC 